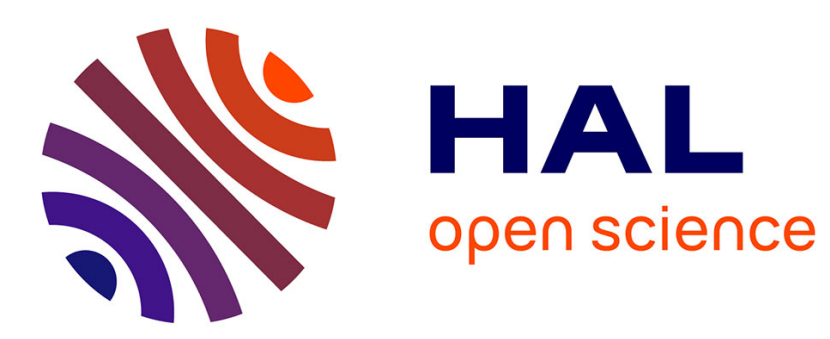

\title{
Composition Functionals in Higher Order Calculus of Variations and Noether's Theorem
}

Gastão S F Frederico, José Vanterler, Ricardo Almeida

\section{To cite this version:}

Gastão S F Frederico, José Vanterler, Ricardo Almeida. Composition Functionals in Higher Order Calculus of Variations and Noether's Theorem. 2020. hal-02955497

\section{HAL Id: hal-02955497 \\ https://hal.science/hal-02955497}

Preprint submitted on 2 Oct 2020

HAL is a multi-disciplinary open access archive for the deposit and dissemination of scientific research documents, whether they are published or not. The documents may come from teaching and research institutions in France or abroad, or from public or private research centers.
L'archive ouverte pluridisciplinaire HAL, est destinée au dépôt et à la diffusion de documents scientifiques de niveau recherche, publiés ou non, émanant des établissements d'enseignement et de recherche français ou étrangers, des laboratoires publics ou privés. 


\title{
Composition Functionals in Higher Order Calculus of Variations and Noether's Theorem
}

\author{
Gastão S. F. Frederico ${ }^{a}$ and J. Vanterler da C. Sousa ${ }^{b}$ and Ricardo Almeida ${ }^{c}$ \\ aFederal University of Ceará, Campus de Russas, Russas, 62900-000 Brazil; \\ b Department of Applied Mathematics, Imecc-Unicamp, 13083-859, Campinas, SP, Brazil; \\ c Center for Research and Development in Mathematics and Applications (CIDMA), \\ Department of Mathematics, University of Aveiro, 3810-193 Aveiro, Portugal
}

\section{ARTICLE HISTORY}

Compiled October 1, 2020

\begin{abstract}
In the present paper we discuss the existence and uniqueness of solution for higherorder calculus of variations problems, involving composition of functionals. Also, higher-order DuBois-Reymond conditions in the Sobolev space $\mathbb{W}^{m, p}\left(\left[t_{1}, t_{2}\right] ; \mathbb{R}\right)$ are proven, both in integral and differential form, and under additional constraints. We consider the higher-order Noether's theorem and discuss invariance conditions for the main problem.
\end{abstract}

\section{KEYWORDS}

Higher-order Noether's theorem; existence and uniqueness, DuBois-Reymond conditions; Euler-Lagrange equations.

\section{Introduction}

In this paper, we consider the following problem of calculus of variations in Lagrange form

$$
\begin{aligned}
\mathcal{J}_{n}^{m}: \Omega & \longrightarrow \mathbb{R} \\
q & \longrightarrow \mathbf{H}\left(\int_{t_{1}}^{t_{2}} L[q]^{m}(t) d t\right) \longrightarrow \min
\end{aligned}
$$

subject to suitable boundary conditions and/or other types of restrictions, where $\Omega$ is a weakly closed subset of $\mathbb{W}^{m, p}\left(\left[t_{1}, t_{2}\right] ; \mathbb{R}\right)$. The Lagrangian $L:\left[t_{1}, t_{2}\right] \times \mathbb{R}^{m+1} \rightarrow \mathbb{R}^{n}$ is assumed to be a $\mathcal{C}^{1}$-function with respect to all its arguments, admissible functions $q(\cdot)$ are assumed to belong to the space $\Omega \subseteq \mathbb{W}^{m, p}\left(\left[t_{1}, t_{2}\right] ; \mathbb{R}\right)$, and $t_{1}<t_{2}$ are fixed in $\mathbb{R}$.

The theory of the calculus of variations over the years has grown exponentially and has gained prominence for its importance and relevance, in particular, involving problems of optimality and physics $[6-8,17,27]$. The history of the calculus of variations drew the attention of a remarkable range of luminary mathematicians, starting with Newton and Leibniz, continuing with the first works by Euler, Lagrange, 
and Laplace. Minimization problems that can be analyzed by calculus of variations serve to characterize the equilibrium configurations of almost all continuous physical systems, varying through elasticity, gravitation, quantum mechanics, among others $[9,14,20,24,25,27,27,29]$. In addition, it should be noted that numerical integration methods, such as the powerful finite element method, are also based on a minimization paradigm $[26,36]$.

On the other hand, it is worth highlighting the varied problems in other contexts like in time scales and in the circle $[4,22,30,31]$. Another field where calculus of variations problems are considered, and has gained a lot of attention in the past decades, is inside the theory of fractional calculus $[1-3,16,18,19,23,28,32]$.

In 2011, Malinowska and Torres [31] considered the problem of calculus of variations for a functional given by

$$
\mathbf{H}\left(\int_{a}^{b} f\left(t, x^{\sigma}(t), x^{\Delta}(t)\right) d \Delta\right)
$$

which is the composition of a certain scalar function $\mathbf{H}$ with the delta integral of a vector valued field $f$. Necessary optimally condition for isoperimetric problem is discussed.

Noether's theorem concerns the connection between a certain type of symmetries and conservation laws in physics [15,40-42]. It was proven by the German mathematician Emmy Noether in her paper "Invariante Variationsprobleme" in 1918 [34]. In the literature, there exists an important range of results involving the Noether's Theorem.

In 2012, Frederico and Torres [15] extended the DuBois-Reymond necessary optimality condition and Noether symmetry theorem to the time delay variational problem. In 2014, Frederico et al. [17] discussed a non-smooth extension of Noether's Theorem, symmetry theorem for variational problems with delayed arguments, and a DuBoisReymond necessary optimality condition.

Christyanti and Wibowo [10] discussed the existence and uniqueness of solutions $\bar{u}$ of the Euler-Lagrange equation in Sobolev space $W^{1, p}$, with Gateaux derivative, for a functional of form

$$
I(u)=\int_{\Omega} F(u, \nabla u, x) d x
$$

where $u: \Omega \rightarrow \mathbb{R}, F \in C^{1}\left(\mathbb{R} \times \mathbb{R}^{n} \times \bar{\Omega}\right)$, and $\nabla u=\frac{\partial u}{\partial x_{i}}, i=1,2, \ldots, n$. Moreover, we find that the minimum $\bar{u}$ for a functional (2) must satisfy the equation

$$
F_{u}(\bar{u}, \nabla \bar{u}, x)-\sum_{i=1}^{n} \frac{\partial}{\partial x_{i}}(F(\bar{u}, \nabla \bar{u}, x))=0
$$

$x \in \bar{\Omega}$, where $F_{u}=\frac{\partial F}{\partial u}$. Other results on the existence and regularity of solutions in the context of calculus of variations can be obtained [11-13].

Note that, when we fix $m=1$, problem (1) reduces to the variational problem 
introduced in [9];

$$
\begin{aligned}
\mathcal{J}_{n}^{1}: \Omega & \longrightarrow \mathbb{R} \\
q & \longrightarrow \mathbf{H}\left(\int_{t_{1}}^{t_{2}} L\left(t, q(t), q^{1}(t)\right)(t) d t\right) \longrightarrow \min
\end{aligned}
$$

Motivated by the works above, this paper aims to discuss some important properties of problem (1) and to contribute significantly to the field. In order to make the understanding and objectives achieved in the paper clear and easy, we highlight the main points below, namely:

(1) We discussed the existence and uniqueness of solutions to the problem of calculus of variations in form (1);

(2) We discussed higher-order DuBois-Reymond conditions for problem (1);

(3) We investigated higher-order Euler-Lagrange equations in integral and differential form, and with constraints like the isoperimetric problem and with holonomic constraint;

(4) We attack the theorem from higher-order Noether and discuss invariance conditions for the problem (1);

The paper is organized as follows. In Section 2 we present some definitions, remarks, and the function spaces important for the development of the paper. In this sense, we attack the problem (1), by discussing the existence and uniqueness of solutions. In Section 3, we investigate higher-order Euler-Lagrange equations in integral and differential forms and DuBois-Reymond conditions for problem (1). In this sense, we investigate some Euler-Lagrange equations under additional constraints, such as the isoperimetric problem and with holomonic constraints. In Section 4, necessary conditions of invariance and higher-order Noether's theorem are discussed.

\section{Existence and uniqueness of solution}

In this section, we start by presenting some important definitions and notes. Then, we will investigate the existence and uniqueness of solutions to the problem of calculus of variations in Lagrange form (1).

Along the work, $1<p<\infty$ and $p^{\prime}$ denotes the adjoint of $p$. Let $\|\cdot\|$ the standard Euclidean norm of $\mathbb{R}^{n}$. For any $1 \leq r \leq \infty$, we denote [5]:

(1) by $\mathrm{L}^{r}:=\mathrm{L}^{r}\left(\left[t_{1}, t_{2}\right] ; \mathbb{R}^{n}\right)$ the usual space of $r$-Lebesgue integrable functions endowed with its usual norm $\|\cdot\|_{\mathrm{L}^{r}}$;

(2) by $\mathcal{C}:=\mathcal{C}\left(\left[t_{1}, t_{2}\right] ; \mathbb{R}\right)$ the standard space of continuous functions and by $\mathcal{C}_{c}^{\infty}:=$ $\mathcal{C}_{c}^{\infty}\left(\left[t_{1}, t_{2}\right] ; \mathbb{R}\right)$ as the standard space of infinitely differentiable functions compactly supported in $\left(t_{1}, t_{2}\right)$;

(3) by $\mathbb{W}^{1, r}:=\mathbb{W}^{1, r}\left(t_{1}, t_{2} ; \mathbb{R}^{n}\right)$ the usual $r$-Sobolev space endowed with its usual norm $\|\cdot\|_{\mathbb{W}^{1, r}} ; \mathbb{W}_{0}^{1, r}=\overline{\mathcal{C}_{c}^{1}\left(\left[t_{1}, t_{2}\right] ; \mathbb{R}^{n}\right)}$.

Let us remind that the compact embedding $\mathbb{W}^{1, r} \hookrightarrow \mathcal{C}$ holds for $1<r \leq+\infty$ (see $[5])$.

For $m \in \mathbb{N}, q^{(i)}(t)$ denotes the $i$-th derivative of $q(t), i=0, \ldots, m$, with $q^{(0)}(t)=$ $q(t)$. For simplicity of notation, we introduce the operator $[\cdot]^{m}$ defined by 


$$
[q]^{m}(t):=\left(t, q(t), q^{(1)}(t), \ldots, q^{(m)}(t)\right) .
$$

Throughout the text, $\partial_{i} L$ denotes the partial derivative of $L$ with respect to its $i$-th argument, $i=1, \ldots, m+2$.

Definition 2.1. Let $\mathcal{J}_{n}^{m}: \Omega \longrightarrow \mathbb{R}$. We say that $\mathcal{J}_{n}^{m}$ is coercive on $\Omega$ if

$$
\mathcal{J}_{n}^{m}[q] \geq \alpha\|q\|+\beta,
$$

for some $\alpha \geq 0$ and $\beta \in \mathbb{R}$.

Remark 1. (1) The coercivity condition ensures that

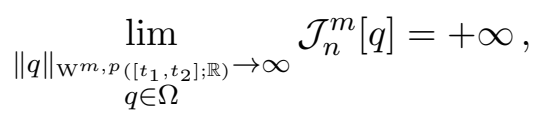

which means that the infimum in problem (1) is finite. This is generally a quite moderate restriction, since many functionals of interest are coercive and this property is easy to check.

(2) It is easy to check that, if each $L_{l}, 1 \leq l \leq n$, satisfies the condition

$$
\alpha\left(\sum_{i=1}^{m}\left|q^{(i)}\right|^{p}\right) \leq L_{l}[q]^{m}(t) \leq \beta\left(|q|^{p}+\sum_{i=1}^{m}\left|q^{(i)}\right|^{p}\right),
$$

with $\alpha, \beta>0$, then $\mathcal{J}_{n}^{m}$ is coercive over $\Omega$. For this, it is sufficient to combine inequality (6) and the Poincaré inequality for functions with higher-order derivatives (Theorem 4.1, [29]).

Definition 2.2. We say that $L$ is regular if each component $L_{l}, 1 \leq l \leq n$, satisfies:

(1) $\mathrm{L}_{l} \in \mathrm{L}^{1}$;

(2) $\frac{\partial \mathrm{L}_{l}}{\partial q} \in \mathrm{L}^{1}$;

(3) $\frac{\partial \mathrm{L}_{l}}{\partial q^{(i)}} \in \mathrm{L}^{p^{\prime}}, 1 \leq i \leq m$

for any $q \in \mathbb{W}^{m, p}$.

Theorem 2.3 (Existence and uniqueness). Let us assume the following hypotheses:

(1) $\left(H_{1}\right) L$ is regular;

(2) $\left(H_{2}\right) \mathcal{J}_{n}^{m}$ is coercive on $\Omega$;

(3) $\left(H_{3}\right) L$ is convex, that is: each component $L_{l}$ is convex on $(\mathbb{R})^{m+1}, 1 \leq l \leq n$, for any $t \in\left[t_{1}, t_{2}\right]$. if

Then there exists a weak minimize (cf. Proposition 3.4) for problem (1). In addition,

(1) $\left(H_{3}^{\prime}\right) L$ is strictly convex, that is: each component $L_{l}$ is strictly convex on $(\mathbb{R})^{m+1}, 1 \leq l \leq n$, for any $t \in\left[t_{1}, t_{2}\right]$,

then this solution is unique. 
Proof. We will prove this theorem in two steps:

Step 1: Existence. Note that $\mathcal{J}_{n}^{m}$ is well-defined because since each $L_{l}, 1 \leq l \leq n$, is regular, $L_{l} \in \mathrm{L}^{1}$ and $\mathcal{J}_{n}^{m}[\cdot]$ exists in $\mathbb{R}$. Let $\left(q_{n}\right)_{n \in \mathbb{N}} \subset \Omega$ be a minimizing sequence satisfying

$$
\mathcal{J}_{n}^{m}\left[q_{n}\right] \longrightarrow \inf _{q \in \Omega} \mathcal{J}_{n}^{m}[q]<+\infty
$$

On the other hand, $\left(H_{2}\right)$ implies that the sequence $\left(q_{n}\right)_{n \in \mathbb{N}}$ is bounded in $\mathbb{W}^{m, p}\left(\left[t_{1}, t_{2}\right] ; \mathbb{R}\right)$. Since $\mathbb{W}^{m, p}$ is a reflexive Banach space, there exists a subsequence of $\left(q_{n}\right)_{n \in \mathbb{N}}$ weekly convergent to $\tilde{q}$ in $\mathbb{W}^{m, p}\left(\left[t_{1}, t_{2}\right] ; \mathbb{R}\right)$. We denote this subsequence by $\left(\tilde{q}_{n}\right)_{n \in \mathbb{N}}$. Then, since $\Omega$ is a weakly closed subset of $\mathbb{W}^{m, p}\left(\left[t_{1}, t_{2}\right] ; \mathbb{R}\right), \tilde{q} \in \Omega$.

Now, from $\left(H_{3}\right)$, we have

$$
\mathcal{J}_{n}^{m}\left[\tilde{q}_{n}\right] \geq \mathcal{J}_{n}^{m}[\tilde{q}]+\sum_{l=1}^{n} \int_{t_{1}}^{t_{2}} \partial_{l} \mathbf{H} \cdot\left(\sum_{i=0}^{m} \partial_{i+2} L_{l}[\tilde{q}]^{m}(t)\left(\tilde{q}_{n}^{(i)}-\tilde{q}^{(i)}\right)\right) d t,
$$

and from the following assumptions:

(1) $\left(H_{1}\right)$ holds;

(2) $\tilde{q}_{n} \longrightarrow \tilde{q}$ in $\mathbb{W}^{1, p}\left(\left[t_{1}, t_{2}\right] ; \mathbb{R}\right) ;$

(3) the compact embedding $\mathbb{W}^{1, p} \hookrightarrow \mathcal{C}$ holds;

one can conclude that, for all $L_{l}, 1 \leq l \leq n$,

(i) $\frac{L_{l}[\tilde{q}]^{m}(t)}{\partial \tilde{q}} \in \mathrm{L}^{1}$ and $\tilde{q}_{n} \longrightarrow \tilde{q}$ in $\mathrm{L}^{\infty}$;

(ii) $\frac{\partial L_{l}[\tilde{q}]^{m}(t)}{\partial \tilde{q}^{(i)}} \in \mathrm{L}^{p^{\prime}}$ and $\tilde{q}_{n}^{(i)} \rightarrow \tilde{q}^{(i)}$ in $\mathrm{L}^{p}, 1 \leq i \leq m$.

Using (7) and we take $n \rightarrow \infty$ in inequality (8), then we have

$$
\inf _{q \in \Omega} \mathcal{J}_{n}^{m}[q] \geq \mathcal{J}_{n}^{m}[\tilde{q}] \in \mathbb{R}
$$

So, we concluded the proof of existence.

Step 2: Uniqueness. Suppose there exists $\tilde{x}_{1}, \tilde{x}_{2} \in \Omega$ such that

$$
\operatorname{Inf}\left\{\mathcal{J}_{n}^{m}[x]: x \in \Omega\right\}=\mathcal{J}_{n}^{m}\left[\tilde{x}_{1}\right]=\mathcal{J}_{n}^{m}\left[\tilde{x}_{2}\right]=\kappa \in \mathbb{R}
$$

and show that this implies $\tilde{x}_{1}=\tilde{x}_{2}$. Let $\tilde{y}=\frac{\tilde{x}_{1}+\tilde{x}_{2}}{2}$ so $\tilde{y} \in \Omega$. Since $L$ is convex, we can say that $\tilde{y}$ is also a minimum of $\mathcal{J}$ because

$$
\kappa \leq \mathcal{J}_{n}^{m}[\tilde{y}] \leq \frac{\mathcal{J}_{n}^{m}\left[\tilde{x}_{1}\right]}{2}+\frac{\mathcal{J}_{n}^{m}\left[\tilde{x}_{2}\right]}{2}=\kappa
$$

Thus, we obtain

$$
\mathbf{H}\left(\int_{t_{1}}^{t_{2}}\left[\frac{L\left[\tilde{x}_{1}\right]^{m}(t)}{2}+\frac{L\left[\tilde{x}_{2}\right]^{m}(t)}{2}-L\left(t, \frac{\tilde{x}_{1}+\tilde{x}_{2}}{2}, \frac{\tilde{x}_{1}^{(1)}+\tilde{x}_{2}^{(1)}}{2}, \cdots, \frac{\tilde{x}_{1}^{(m)}+\tilde{x}_{2}^{(m)}}{2}\right)\right] d t\right)=0
$$


The convexity of $L$ ensures that the integrant is non-negative. As the integral is null, so the only possibility is

$$
\frac{L\left[\tilde{x}_{1}\right]^{m}(t)}{2}+\frac{L\left[\tilde{x}_{2}\right]^{m}(t)}{2}-L\left(t, \frac{\tilde{x}_{1}+\tilde{x}_{2}}{2}, \frac{\tilde{x}_{1}^{(1)}+\tilde{x}_{2}^{(1)}}{2}, \cdots, \frac{\tilde{x}_{1}^{(m)}+\tilde{x}_{2}^{(m)}}{2}\right)=0
$$

on the interval $\left[t_{1}, t_{2}\right]$.

We now use $\left(H_{3}^{\prime}\right)$ to get that $\tilde{x}_{1}^{(i)}=\tilde{x}_{2}^{(i)}, 0 \leq i \leq m$, on $\left[t_{1}, t_{2}\right]$, as asserted. The proof is complete.

\section{Higher-order Euler-Lagrange equation and DuBois-Reymond condition}

In this section, we are going to attack the higher-order Euler-Lagrange theorem in integral and differential form. In this sense, we also discuss the Euler-Lagrange equation with constraints such as the isoperimetric problem and when in presence of holonomic constraints. Finally, the DuBois-Reymond condition is discussed.

Definition 3.1. [Gâteaux-differential] The first-order Gâteaux-differential of $\mathcal{J}_{n}^{m}$ at some $q \in \Omega \subseteq \mathbb{W}^{m, p}\left(\left[t_{1}, t_{2}\right] ; \mathbb{R}\right)$ is defined by

$$
\mathcal{D} \mathcal{J}_{n}^{m}[q](h):=\lim _{\epsilon \rightarrow 0} \frac{\mathcal{J}_{n}^{m}[q+\epsilon h]-\mathcal{J}_{n}^{m} J[q]}{\epsilon},
$$

for all $h \in \Omega, \epsilon \in \mathbb{R}$, provided that the right-hand side term exists.

Theorem 3.2. The differential of $\mathcal{J}_{n}^{m}[(\cdot)]$ at $q(\cdot) \in \Omega$ is given by

$$
D \mathcal{J}_{n}^{m}[q](h)=\sum_{l=1}^{n} \int_{t_{1}}^{t_{2}} \partial_{l} \mathbf{H} \cdot\left(\sum_{i=0}^{m} \partial_{i+2} L_{l}[q]^{m}(t)\right) h^{(i)}(t) d t
$$

for all $h \in \Omega$, where $\partial_{l} \mathbf{H}$ represents the partial derivative of $\mathbf{H}$ with respect to its lth argument, evaluate at

$$
\int_{t_{1}}^{t_{2}} L[q]^{m}(t) d t \in \mathbb{R}^{n}
$$

Proof. We obtain equation (10) by direct computations with help of a Taylor expansion.

Definition 3.3. [Extremal of (1)] We say that $q$ is an extremal (or critical point) of the functional (1) if for any $h(\cdot) \in \Omega$ such that $h^{(i)}\left(t_{1}\right)=h^{(i)}\left(t_{2}\right)=0, i=0, \ldots, m-1$, the following equation holds:

$$
\left.\mathcal{D} \mathcal{J}_{n}^{m}[q](h)\right|_{\epsilon=0}=0 .
$$

We have the well known result of calculus of variations: 
Proposition 3.4. If $q(\cdot) \in \Omega$ is a weak minimizer of functional $\mathcal{J}_{n}^{m}$, i.e.

$$
\mathcal{J}_{n}^{m}[q] \leq \mathcal{J}_{n}^{m}[q+h]
$$

for all $h(\cdot) \in \mathcal{C}_{c}^{\infty}$ with $\|h\|_{C^{1}([a, b])}<\delta, 0<\delta \ll 1$, then $q$ is an extremal of functional (1), where

$$
\|h\|_{C^{m}\left(\left[t_{1}, t_{2}\right]\right)}:=\max _{t \in\left[t_{1}, t_{2}\right]}|h(t)|+\sum_{i=1}^{m} \max _{t \in\left[t_{1}, t_{2}\right]}\left|h^{(i)}(t)\right| .
$$

Clearly, a weak minimizer is also an extremal, but the opposite is not true. For example, local minimal and saddle points may also occur as solutions.

Theorem 3.5 (Higher-order Euler-Lagrange equations in integral form). If $q(\cdot) \in \Omega$ is an extremal of functional (1), then $q(\cdot)$ satisfies the following higher-order EulerLagrange integral equations:

$$
\sum_{l=1}^{n} \partial_{l} \mathbf{H} \cdot\left(\sum_{i=0}^{m}(-1)^{m-i-1}(\underbrace{\int_{t_{1}} t \int_{t_{1}}^{s_{1}} \ldots \int_{t_{1}}^{s_{m-i-1}}}_{m-i \text { times }}\left(\partial_{i+2} L_{l}[q]^{m}(t)\right) d s_{m-i} \ldots d s_{2} d s_{1})\right)
$$

where $p(t)$ is a polynomial of order $m-1$, i.e., $p(t)=c_{0}+c_{1} t+\cdots+c_{m-1} t^{m-1}$, for some constants $c_{i} \in \mathbb{R}, i=0, \ldots, m-1$.

Proof. Assume that $q(\cdot) \in \Omega$ is an extremal of functional (1). According to the Theorem 3.2 and Definition 3.3, for any $h(\cdot) \in \Omega$ such that $h^{(i)}\left(t_{1}\right)=h^{(i)}\left(t_{2}\right)=0, i=$ $0, \ldots, m-1$, we have

$$
\sum_{l=1}^{n} \int_{t_{1}}^{t_{2}} \partial_{l} \mathbf{H} \cdot\left(\sum_{i=0}^{m} \partial_{i+2} L_{l}[q]^{m}(t)\right) h^{(i)}(t) d t=0 .
$$

By repeated integration by parts, yields

$$
\begin{aligned}
& \sum_{l=1}^{n}\left(\sum_{i=0}^{m} \int_{t_{1}}^{t_{2}} \partial_{l} \mathbf{H} \cdot\left(\partial_{i+2} L_{l}[q]^{m}(t)\right) h^{(i)}(t) d t\right) \\
= & \sum_{l=1}^{n} \sum_{i=0}^{m}\left\{\left[\sum_{j=1}^{m-i}(-1)^{j+1} h^{(i+j-1)}(t)(\underbrace{\int_{t_{1}}^{t} \int_{t_{1}}^{s_{1}} \cdots \int_{t_{1}}^{s_{j-1}}}_{j \text { times }} \partial_{l} \mathbf{H} \cdot\left(\partial_{i+2} L_{l}[q]^{m}\left(s_{j}\right)\right) d s_{j} \ldots d s_{2} d s_{1})\right]_{t_{1}}^{t_{2}}\right. \\
+ & \left.(-1)^{i} \int_{t_{1}}^{t_{2}} h^{(m)}(t)(\underbrace{\int_{t_{1}}^{t} \int_{t_{1}}^{s_{1}} \ldots \int_{t_{1}}^{s_{m-i-1}}}_{m-i \text { times }} \partial_{l} \mathbf{H} \cdot\left(\partial_{i+2} L_{l}[q]^{m}\left(s_{m-i}\right)\right) d s_{m-i} \ldots d s_{2} d s_{1}) d t\right\} .
\end{aligned}
$$


Because $h^{(i)}\left(t_{1}\right)=0=h^{(i)}\left(t_{2}\right), i=0, \ldots, m-1$, the terms without integral sign in the right-hand sides of identities (13) vanish. Therefore, equation (10) becomes

$$
\begin{aligned}
& 0= \\
& \sum_{l=1}^{n} \int_{t_{1}}^{t_{2}} h^{(m)}(t)\left[\sum_{i=0}^{m}(-1)^{i}(\underbrace{\int_{t_{1}}^{t} \int_{t_{1}}^{s_{1}} \ldots \int_{t_{1}}^{s_{m-i-1}}}_{m-i \text { times }} \partial_{l} \mathbf{H} \cdot\left(\partial_{i+2} L_{l}[q]^{m}\left(s_{m-i}\right)\right) d s_{m-i} \ldots d s_{2} d s_{1})\right] d t .
\end{aligned}
$$

Applying the higher-order DuBois-Reymond Lemma [25,36], one arrives to equation (11).

Corollary 3.6 (Higher-order Euler-Lagrange equations in differential form). If $q(\cdot) \in$ $\Omega$ is an extremal of functional (1), then

$$
\sum_{l=1}^{n} \partial_{l} \mathbf{H} \cdot\left(\sum_{i=0}^{m}(-1)^{i} \frac{d^{i}}{d t^{i}} \partial_{i+2} L_{l}[q]^{m}(t)\right)=0
$$

for all $t \in\left[t_{1}, t_{2}\right]$.

Proof. We obtain (14) applying the derivative of order $m$ to (11).

In practice, we are going to use more Corollary 3.6 than Theorem 3.5. There are important general properties of Euler-Lagrange systems based on the symmetry of the Lagrangian. The most important symmetry result is the Noether's Theorem (cf. Theorem 4.5).

Remark 2. If $q(\cdot)$ is an extremal of the functional (3), one obtains from equation (3.6) for $m=1$ that

$$
\sum_{l=1}^{n} \partial_{l} \mathbf{H} \cdot\left(\partial_{2} L_{l}(t, q(t), \dot{q}(t))-\frac{d}{d t} \partial_{3} L_{l}(t, q(t), \dot{q}(t))\right)=0 .
$$

In [9], the authors discussed two important cases of problem (1): for $\mathrm{m}=1$, the product functional and the ratio or quotient functional for $n=2$. Here, these two cases can be easily generalized in our problem (1) for $n=2$.

(1) The product functional. In this case

$$
\mathcal{J}_{2}^{m}(q)=\left(\int_{t_{1}}^{t_{2}} L_{1}[q]^{m} d t\right)\left(\int_{t_{1}}^{t_{2}} L_{2}[q]^{m} d t\right)
$$

and we can derive the Euler-Lagrange equation associated with functional (16) using Corollary 3.6, given by 


$$
\begin{aligned}
\left(\sum_{i=0}^{m}(-1)^{i} \frac{d^{i}}{d t^{i}} \partial_{i+2} L_{1}[q]^{m}(t)\right) & \left(\int_{t_{1}}^{t_{2}} L_{2}[q]^{m} d t\right) \\
& +\left(\sum_{i=0}^{m}(-1)^{i} \frac{d^{i}}{d t^{i}} \partial_{i+2} L_{2}[q]^{m}(t)\right)\left(\int_{t_{1}}^{t_{2}} L_{1}[q]^{m} d t\right)=0 .
\end{aligned}
$$

(2) The quotient functional.

Now, the quotient functional is

$$
Q_{2}^{m}=\frac{\int_{t_{1}}^{t_{2}} L_{1}[q]^{m} d t}{\int_{t_{1}}^{t_{2}} L_{2}[q]^{m} d t}
$$

and the Euler-Lagrange equation associated with functional (17) using Corollary 3.6 , given by

$$
\begin{aligned}
\left(\sum_{i=0}^{m}(-1)^{i} \frac{d^{i}}{d t^{i}} \partial_{i+2} L_{1}[q]^{m}(t)\right) & \left(\int_{t_{1}}^{t_{2}} L_{2}[q]^{m} d t\right) \\
& =\left(\sum_{i=0}^{m}(-1)^{i} \frac{d^{i}}{d t^{i}} \partial_{i+2} L_{2}[q]^{m}(t)\right)\left(\int_{t_{1}}^{t_{2}} L_{1}[q]^{m} d t\right) .
\end{aligned}
$$

\subsection{DuBois-Reymond condition}

Associated to a given function $q(\cdot) \in \Omega$, it is useful to introduce the following quantity, given by

$$
\varphi^{j}=\sum_{i=0}^{m-j}(-1)^{i} \frac{d^{i}}{d t^{i}} \partial_{i+j+2} L[q]^{m}(t)
$$

for $t \in\left[t_{1}, t_{2}\right]$, where $j=0, \ldots, m$. These operators are useful for our purposes because of the following property

$$
\frac{d}{d t} \varphi^{j}=\partial_{j+1} L[q]^{m}(t)-\varphi^{j-1}
$$

for $t \in\left[t_{1}, t_{2}\right]$, where $j=1, \ldots, m$.

We are now in conditions to prove a higher-order DuBois-Reymond optimality condition for variational problem (1).

Theorem 3.7 (Higher-order DuBois-Reymond condition). If $q \in \Omega$ is an extremal of 
functional (1), then

$$
\sum_{l=1}^{n} \partial_{l} \mathbf{H} \cdot\left(\frac{d}{d t}\left(L_{l}[q]^{m}(t)-\sum_{j=1}^{m} \varphi^{j} q^{(j)}(t)\right)\right)=\sum_{l=1}^{n} \partial_{l} \mathbf{H} \cdot\left(\partial_{1} L_{l}[q]^{m}(t)\right)
$$

for $t_{1} \leq t \leq t_{2}$, where $\varphi^{j}$ is given by (19).

Proof. We derive equation (21) as follows

$$
\begin{aligned}
\frac{d}{d t} \mathbf{H} & \left(\int_{t_{1}}^{t_{2}}\left(L[q]^{m}(t)-\sum_{j=1}^{m} \varphi^{j} q^{(j)}(t)\right) d t\right)=\sum_{l=1}^{n} \int_{t_{1}}^{t_{2}} \partial_{l} \mathbf{H} \cdot\left(\partial_{1} L_{l}[q]^{m}(t)\right) \\
& \left.+\sum_{j=0}^{m} \partial_{j+2} L_{l}[q]^{m}(t) q^{(j+1)}(t)-\sum_{j=1}^{m}\left(\frac{d \varphi^{j}}{d t} q^{(j)}(t)+\varphi^{j} q^{(j+1)}(t)\right)\right) d t
\end{aligned}
$$

From equations (20) and (22), yields

$$
\begin{array}{r}
\frac{d}{d t} \mathbf{H}\left(\int_{t_{1}}^{t_{2}}\left(L[q]^{m}(t)-\sum_{j=1}^{m} \varphi^{j} q^{(j)}(t)\right) d t\right) \\
=\sum_{l=1}^{n} \int_{t_{1}}^{t_{2}} \partial_{l} \mathbf{H} \cdot\left(\partial_{1} L_{l}[q]^{m}(t)+\sum_{j=0}^{m} \partial_{j+2} L_{l}[q]^{m}(t) q^{(j+1)}(t)\right. \\
\left.-\sum_{j=1}^{m}\left(\left(\partial_{j+1} L_{l}[q]^{m}(t)-\varphi^{j-1}\right) q^{(j)}(t)+\varphi^{j} q^{(j+1)}(t)\right)\right) d t .
\end{array}
$$

We now simplify the second term on the right-hand side of (23):

$$
\begin{aligned}
& \sum_{j=1}^{m}\left(\left(\partial_{j+1} L_{l}[q]^{m}(t)-\varphi^{j-1}\right) q^{(j)}(t)+\varphi^{j} q^{(j+1)}(t)\right) \\
= & \sum_{j=0}^{m-1}\left(\left(\partial_{j+2} L_{l}[q]^{m}(t)-\varphi^{j}\right) q^{(j+1)}(t)+\varphi^{j+1} q^{(j+2)}(t)\right) \\
= & \sum_{j=0}^{m-1} \partial_{j+2} L_{l}[q]^{m}(t) q^{(j+1)}-\varphi^{0} q^{(1)}(t)+\varphi^{m} q^{(m+1)}(t) .
\end{aligned}
$$

Substituting the equation (24) into equation (23) and using the higher-order EulerLagrange equations (14), and since by definition,

$$
\varphi^{m}=\partial_{m+2} L_{l}[q]^{m}(t)
$$


and

$$
\varphi^{0}=\sum_{i=0}^{m}(-1)^{i} \frac{d^{i}}{d t^{i}} \partial_{i+2} L_{l}[q]^{m}(t)
$$

we obtain the intended result, that is,

$$
\begin{aligned}
& \frac{d}{d t} \mathbf{H}\left(\int_{t_{1}}^{t_{2}}\left(L[q]^{m}(t)-\sum_{j=1}^{m} \varphi^{j} q^{(j)}(t)\right) d t\right) \\
= & \sum_{l=1}^{n} \int_{t_{1}}^{t_{2}} \partial_{l} \mathbf{H} \cdot\left[\partial_{1} L_{l}[q]^{m}(t)+\partial_{m+2} L_{l}[q]^{m}(t) q^{(m+1)}+\varphi^{0} q^{(1)}(t)-\varphi^{m} q^{(m+1)}(t)\right] d t \\
= & \sum_{l=1}^{n} \int_{t_{1}}^{t_{2}} \partial_{l} \mathbf{H} \cdot \partial_{1} L_{l}[q]^{m}(t) d t .
\end{aligned}
$$

We can easily prove the following result:

Corollary 3.8 (DuBois-Reymond condition for Problem (3)). If $q(\cdot) \in \Omega$ is an extremal of functional (3) subject to some suitable boundary conditions, then the following conditions are satisfied:

$$
\sum_{l=1}^{n} \partial_{l} \mathbf{H} \cdot\left(\frac{d}{d t}\left(L_{l}(t, q(t), \dot{q}(t))-\partial_{3} L_{l}(t, q(t), \dot{q}(t) \dot{q}(t))\right)=\sum_{l=1}^{n} \partial_{l} \mathbf{H} \cdot\left(\partial_{1} L_{l}(t, q(t), \dot{q}(t))\right) .\right.
$$

\subsection{Euler-Lagrange equation under constraints}

In this section we seek necessary conditions of optimization, subject to some additional constraints. Consider the functional

$$
\begin{aligned}
\mathcal{I}_{n}^{m}: \Omega & \longrightarrow \mathbb{R} \\
q & \longrightarrow \mathbf{H}\left(\int_{t_{1}}^{t_{2}} M[q]^{m}(t) d t\right)
\end{aligned}
$$

where $M:\left[t_{1}, t_{2}\right] \times \mathbb{R}^{m+1} \rightarrow \mathbb{R}^{n}$ is of class $\mathcal{C}^{1}$, and the equation

$$
g(t, q(t))=0, \quad t \in\left[t_{1}, t_{2}\right]
$$

where $g:\left[t_{1}, t_{2}\right] \times \mathbb{R}^{2} \rightarrow \mathbb{R}$ is of class $\mathcal{C}^{1}$ and $q:=\left(q_{1}, q_{2}\right)$.

Theorem 3.9 (Isoperimetric problem). Let $q(\cdot) \in \Omega$ be an extremal of problem (1), subject to the constraint $\mathcal{I}_{n}^{m}[q]=C$, where $C$ is a fixed real number and $\mathcal{I}_{n}^{m}$ is the functional given in (26). If

$$
\sum_{l=1}^{n} \partial_{l} \mathbf{H} \cdot\left(\sum_{i=0}^{m}(-1)^{i} \frac{d^{i}}{d t^{i}} \partial_{i+2} M_{l}[q]^{m}(t)\right) \neq 0,
$$


at some point $t \in\left[t_{1}, t_{2}\right]$, then there exists a Lagrange multiplier $\lambda \in \mathbb{R}$ such that

$$
\sum_{l=1}^{n} \partial_{l} \mathbf{H} \cdot\left(\sum_{i=0}^{m}(-1)^{i} \frac{d^{i}}{d t^{i}} \partial_{i+2} N_{l}[q]^{m}(t)\right)=0,
$$

for all $t \in\left[t_{1}, t_{2}\right]$, where function $N$ is the Hamiltonian, $N=L+\lambda M$.

Proof. Define functions $j, i: B_{R}(0,0) \rightarrow \mathbb{R}$ by

$$
j\left(\epsilon_{1}, \epsilon_{2}\right)=\mathcal{J}_{n}^{m}\left[q+\epsilon_{1} h_{1}+\epsilon_{2} h_{2}\right] \quad \text { and } \quad i\left(\epsilon_{1}, \epsilon_{2}\right)=\mathcal{I}_{n}^{m}\left[q+\epsilon_{1} h_{1}+\epsilon_{2} h_{2}\right]-C,
$$

where $R>0$ is the radius of the open ball and functions $h_{k}(\cdot) \in \Omega$ are such that $h_{k}^{(i)}\left(t_{1}\right)=h_{k}^{(i)}\left(t_{2}\right)=0, i=0, \ldots, m-1$ and $k=1,2$. Since

$$
\partial_{2} i(0,0)=\int_{t_{1}}^{t_{2}} \sum_{l=1}^{n} \partial_{l} \mathbf{H} \cdot\left(\sum_{i=0}^{m}(-1)^{i} \frac{d^{i}}{d t^{i}} \partial_{i+2} M_{l}[q]^{m}(t)\right) h_{2}(t) d t
$$

by assumption (28), we conclude that there exists a function $h_{2}$ satisfying $\partial_{2} i(0,0) \neq 0$. Then, applying the Implicit Function Theorem, there exists an open set $U \subseteq \mathbb{R}$ and a unique differentiable function $\psi: U \rightarrow \mathbb{R}$ such that $\psi(0)=0$ and $i\left(\epsilon_{1}, \psi\left(\epsilon_{1}\right)\right)=0$, for all $\epsilon_{1} \in U$. In other words, there exists a family of variations $q+\epsilon_{1} h_{1}+\psi\left(\epsilon_{1}\right) h_{2}$ satisfying the integral constraint (26), where $\epsilon_{1}$ is a free parameter.

Once $(0,0)$ is a minimum of $j$, subject to the restriction $i(\cdot, \cdot)=0$, and since $\nabla i(0,0) \neq(0,0)$, by the Lagrange multiplier rule, there exists a real number $\lambda$ such that $\nabla(j+\lambda i)(0,0)=(0,0)$. In particular, $\partial_{1}(j+\lambda i)(0,0)=0$. Doing these computations, we derive equation (29).

In particular, when $m=n=1$ and $\mathbf{H}=\mathrm{Id}$, we obtain the classical result for isoperimetric variational problems (see [6]):

If function $q(\cdot)$ is an extremal of $(1)$, subject to the constraint $\mathcal{I}_{1}^{1}[q]=C$, and if

$$
\partial_{2} M[q]^{1}(t)-\frac{d}{d t} \partial_{3} M[q]^{1}(t) \neq 0
$$

at some point $t \in\left[t_{1}, t_{2}\right]$, then there exists a Lagrange multiplier $\lambda \in \mathbb{R}$ such that

$$
\partial_{2} N[q]^{1}(t)-\frac{d}{d t} \partial_{3} N[q]^{1}(t) \neq 0
$$

for all $t \in\left[t_{1}, t_{2}\right]$, where function $N$ is the Hamiltonian, $N=L+\lambda M$.

The next result generalizes Theorem 3.9, by dropping assumption (28).

Theorem 3.10 (Isoperimetric problem-general form). Let $q(\cdot) \in \Omega$ be an extremal of (1), subject to the constraint $\mathcal{I}_{n}^{m}[q]=C$. Then, there exists two real numbers $\lambda_{0}, \lambda$, not both zero, such that

$$
\sum_{l=1}^{n} \partial_{l} \mathbf{H} \cdot\left(\sum_{i=0}^{m}(-1)^{i} \frac{d^{i}}{d t^{i}} \partial_{i+2} N_{l}[q]^{m}(t)\right)=0
$$

for all $t \in\left[t_{1}, t_{2}\right]$, where $N=\lambda_{0} L+\lambda M$. 
Proof. If assumption (28) holds, then the results follows from Theorem 3.9 and choosing $\lambda_{0}=1$ and $\lambda$ given by Theorem 3.9. Otherwise, choose $\left(\lambda_{0}, \lambda\right)=(1,0)$.

Theorem 3.11 (Holonomic constraint). Let $q(\cdot) \in \Omega^{2}$ be an extremal of functional $\mathcal{J}_{n, 2}^{m}$ given by

$$
\begin{aligned}
\mathcal{J}_{n, 2}^{m}: \Omega^{2} & \longrightarrow \mathbb{R} \\
q & \longrightarrow \mathbf{H}\left(\int_{t_{1}}^{t_{2}} L[q]_{2}^{m}(t) d t\right)
\end{aligned}
$$

where

$$
[q]_{2}^{m}(t):=\left(t, q_{1}(t), q_{2}(t), q_{1}^{(1)}(t), q_{2}^{(1)}(t), \ldots, q_{1}^{(m)}(t), q_{2}^{(m)}(t)\right)
$$

and $L:\left[t_{1}, t_{2}\right] \times \mathbb{R}^{2 m+2} \rightarrow \mathbb{R}^{n}$ is a $\mathcal{C}^{1}$-function, subject to the constraint (27). If $\partial_{3} g(t, q(t)) \neq 0$, for all $t \in\left[t_{1}, t_{2}\right]$, then there exists a function $\lambda:\left[t_{1}, t_{2}\right] \rightarrow \mathbb{R}$ such that

$$
\sum_{l=1}^{n} \partial_{l} \mathbf{H} \cdot\left(\sum_{i=0}^{m}(-1)^{i} \frac{d^{i}}{d t^{i}} \partial_{2 i+2} L_{l}[q]_{2}^{m}(t)\right)+\lambda(t) \partial_{2} g(t, q(t))=0
$$

and

$$
\sum_{l=1}^{n} \partial_{l} \mathbf{H} \cdot\left(\sum_{i=0}^{m}(-1)^{i} \frac{d^{i}}{d t^{i}} \partial_{2 i+3} L_{l}[q]_{2}^{m}(t)\right)+\lambda(t) \partial_{3} g(t, q(t))=0,
$$

for all $t \in\left[t_{1}, t_{2}\right]$.

Proof. Let $q+\epsilon h$ be a variation of the optimal solution, where $h=\left(h_{1}, h_{2}\right)$ with $h^{(i)}\left(t_{1}\right)=h^{(i)}\left(t_{2}\right)=(0,0), i=0, \ldots, m-1$, satisfying equation $(27)$, that is, $g(t, q(t)+$ $\epsilon h(t))=0$. Differentiating with respect to $\epsilon$ and putting $\epsilon=0$, we get

$$
\partial_{2} g(t, q(t)) h_{1}(t)+\partial_{3} g(t, q(t)) h_{2}(t)=0, \quad t \in\left[t_{1}, t_{2}\right] .
$$

Define function $\lambda(\cdot)$ by the formula

$$
\lambda(t):=-\frac{\sum_{l=1}^{n} \partial_{l} \mathbf{H} \cdot\left(\sum_{i=0}^{m}(-1)^{i} \frac{d^{i}}{d t^{i}} \partial_{2 i+3} L_{l}[q]_{2}^{m}(t)\right)}{\partial_{3} g(t, q(t))}
$$

with $t \in\left[t_{1}, t_{2}\right]$. Thus, equation (31) is proven. Regarding equation (30), first observe that, since $q(\cdot)$ is an extremal of functional $\mathcal{J}_{n, 2}^{m}$, the first variation of the functional must vanish:

$$
\int_{t_{1}}^{t_{2}} \sum_{l=1}^{n} \partial_{l} \mathbf{H} \cdot \sum_{i=0}^{m}\left[\left(\partial_{2 i+2} L_{l}[q]_{2}^{m}(t)\right) h_{1}^{(i)}(t)+\left(\partial_{2 i+3} L_{l}[q]_{2}^{m}(t)\right) h_{2}^{(i)}(t)\right] d t=0 .
$$


Performing integration by parts, we obtain

$$
\begin{aligned}
\int_{t_{1}}^{t_{2}} \sum_{l=1}^{n} \partial_{l} \mathbf{H} \cdot \sum_{i=0}^{m}\left[\left((-1)^{i} \frac{d^{i}}{d t^{i}} \partial_{2 i+2} L_{l}[q]_{2}^{m}(t)\right) h_{1}(t)\right. \\
\left.+\left((-1)^{i} \frac{d^{i}}{d t^{i}} \partial_{2 i+3} L_{l}[q]_{2}^{m}(t)\right) h_{2}(t)\right] d t=0
\end{aligned}
$$

Using the equations (32) and (33), we deduce that

$$
\sum_{l=1}^{n} \partial_{l} \mathbf{H} \cdot\left(\sum_{i=0}^{m}(-1)^{i} \frac{d^{i}}{d t^{i}} \partial_{2 i+3} L_{l}[q]_{2}^{m}(t)\right) h_{2}(t)=\lambda(t) \partial_{2} g(t, q(t)) h_{1}(t),
$$

and so equation (34) becomes

$$
\int_{t_{1}}^{t_{2}} \sum_{l=1}^{n} \partial_{l} \mathbf{H} \cdot\left[\sum_{i=0}^{m}\left((-1)^{i} \frac{d^{i}}{d t^{i}} \partial_{2 i+2} L_{l}[q]_{2}^{m}(t)\right)+\lambda(t) \partial_{2} g(t, q(t))\right] h_{1}(t) d t=0 .
$$

From the arbitrariness of function $h_{1}$, we prove equation (30).

Again, when $m=n=1$ and $\mathbf{H}=\mathrm{Id}$, we obtain the classical result for holonomic variational problems (see [6]):

If function $q(\cdot)$ is an extremal of functional $\mathcal{J}_{1,2}^{1}$, subject to the constraint (27), and if $\partial_{3} g(t, q(t)) \neq 0$, for all $t \in\left[t_{1}, t_{2}\right]$, then there exists a function $\lambda:\left[t_{1}, t_{2}\right] \rightarrow \mathbb{R}$ such that

$$
\partial_{2} L[q]_{2}^{1}(t)-\frac{d}{d t} \partial_{4} L[q]_{2}^{1}(t)+\lambda(t) \partial_{2} g(t, q(t))=0
$$

and

$$
\partial_{3} L[q]_{2}^{1}(t)-\frac{d}{d t} \partial_{5} L[q]_{2}^{1}(t)+\lambda(t) \partial_{3} g(t, q(t))=0
$$

for all $t \in\left[t_{1}, t_{2}\right]$.

\section{Noether's theorem}

In the remarkable paper written in 1918 [34], E. Noether proved that there is a oneto-one correspondence between symmetry groups of a variational problem and conservation laws of its Euler-Lagrange equations.

\subsection{Constant of motion/conservation law}

The following definition is essential to our goal to prove Noether's theorem.

Definition 4.1 (Group of symmetries). For any $s \in \mathbb{R}$, let $\psi(s, \cdot): \mathbb{W}^{m, p}(\mathbb{R}) \rightarrow$ $\mathbb{W}^{m, p}(\mathbb{R})$ be a diffeomorphism. We say that $\Psi=\{\psi(s, \cdot)\}_{s \in \mathbb{R}}$ is a one parameter group of diffeomorphisms of $\mathbb{W}^{m, p}(\mathbb{R})$ if it satisfies: 
(1) $\forall s \in \mathbb{R}$, the map $\Psi$ is invertible, and $\Psi^{-1} \in \mathbb{W}^{m, p}(\mathbb{R})$;

(2) $\psi(0, \cdot)=I d_{\mathbb{W} m, p(\mathbb{R})}$;

(3) $\forall s, s^{\prime} \in \mathbb{R}, \psi(s, \cdot) \circ \psi\left(s^{\prime}, \cdot\right)=\psi\left(s+s^{\prime}, \cdot\right)$.

Usual examples of one parameter groups of diffeomorphisms are given by translations in a given direction $\eta$

$$
\psi: q \longmapsto q+\epsilon \eta, q \in \mathbb{R}
$$

and rotations of angle $\omega$

$$
\psi: q \longmapsto q e^{i \epsilon \omega}, q \in \mathbb{C}
$$

Before we can discuss Noether's theorem in detail, we need to discuss what we mean by a symmetry of a system. What we use to describe the system are the equations of motion, so it is natural to say that a symmetry transformation of a system is a transformation of the dependent and independent variables that leaves the explicit form of the equations of motion unchanged. To arrive at this, we introduce the following definition:

Definition 4.2 (Invariance of problem (1)). Functional (1) is said to be $s$-invariant under the action of one parameter group of diffeomorphisms $\Psi_{i}=\left\{\psi_{i}(s, \cdot)\right\}_{s \in \mathbb{R}} \in$ $\mathbb{W}^{m, p}(\mathbb{R}), i=1,2$, if it satisfies for any extremals

$$
\begin{aligned}
& \mathbf{H}\left(\int_{t_{a}}^{t_{b}} L\left(t, q(t), q^{(1)}(t), \ldots, q^{(m)}(t)\right) d t\right) \\
= & \mathbf{H}\left(\int_{\psi_{1}\left(s, t_{a}\right)}^{\psi_{1}\left(s, t_{b}\right)} L\left(\psi_{1}(s, t), \psi_{2}(s, q(t)), \frac{d \psi_{2}(s, q(t))}{d \psi_{1}(s, t)}, \ldots, \frac{d^{m} \psi_{2}(s, q(t))}{d \psi_{1}^{m}(s, t)}\right) \frac{d \psi_{1}}{d t}(s, t) d t\right)
\end{aligned}
$$

for any sub-interval $\left[t_{a}, t_{b}\right] \subseteq[a, b]$.

Notice that, from Definition 4.1, we have that

$$
\int_{\psi_{1}\left(s, t_{a}\right)}^{\psi_{1}\left(s, t_{b}\right)} L\left(\psi_{1}(s, t), \psi_{2}(s, q(t)), \frac{d \psi_{2}(s, q(t))}{d \psi_{1}(s, t)}, \ldots, \frac{d^{m} \psi_{2}(s, q(t))}{d \psi_{1}^{m}(s, t)}\right) \frac{d \psi_{1}}{d t}(s, t) d t<\infty
$$

consequently (35) is well-defined.

Remark 3. In (35), the expressions $\frac{d^{i} \psi_{2}(s, q(t))}{d \psi_{1}^{i}(s, q(t))}, i=1, \ldots, m$, are to be interpreted as

$$
\frac{d \psi_{2}(s, q(t))}{d \psi_{1}(s, t)}=\frac{\frac{d \psi_{2}(s, q(t))}{d t}}{\frac{d \psi_{1}(s, t)}{d t}}
$$


and

$$
\frac{d^{i} \psi_{2}(s, q(t))}{d \psi_{1}^{i}(s, t)}=\frac{\frac{d}{d t}\left(\frac{d^{i-1} \psi_{2}(s, q(t))}{d \psi_{1}^{i-1}(s, t)}\right)}{\frac{d \psi_{1}(s, t)}{d t}},(i=2, \ldots, m)
$$

The next Lemma is very useful to check for $s$-invariance. It is also useful to determine the $s$-invariance transformations.

Lemma 4.3 (Necessary condition of invariance for problem (1)). If functional (1) is invariant in the sense of Definition 4.2, then

$$
\sum_{l=1}^{n} \partial_{l} \mathbf{H} \cdot\left(\frac{\partial L_{l}[q]^{m}(t)}{\partial t} \frac{\partial \psi_{1}}{\partial s}(0, t)+\sum_{i=0}^{m} \frac{\partial L_{l}[q]^{m}(t)}{\partial q^{(i)}(t)} \rho^{i}(t)+L_{l}[q]^{m}(t) \frac{d}{d t} \frac{\partial \psi_{1}}{\partial s}(0, t)\right)=0
$$

where

$$
\left\{\begin{array}{l}
\rho^{0}(t)=\frac{\partial \psi_{2}}{\partial s}(0, q(t)) \\
\rho^{i}(t)=\frac{d \rho^{i-1}}{d t}(t)-q^{(i)} \frac{d}{d t} \frac{\partial \psi_{1}}{\partial s}(0, t), i=1, \ldots, m
\end{array}\right.
$$

Proof. Differentiating (35) with respect to $s$ and substituting $s=0$, one obtains

$$
\begin{aligned}
\int_{t_{a}}^{t_{b}} \sum_{l=1}^{n} \partial_{l} \mathbf{H} \cdot\left(\frac{\partial L_{l}[q]^{m}(t)}{\partial t} \frac{\partial \psi_{1}}{\partial s}(0, t)+\left.\sum_{i=0}^{m} \frac{\partial L_{l}[q]^{m}(t)}{\partial q^{(i)}(t)} \frac{\partial}{\partial s} \frac{d^{i} \psi_{2}(s, q(t))}{d \psi_{1}^{i}(s, t)}\right|_{s=0}\right. \\
\left.+L_{l}[q]^{m}(t) \frac{d}{d t} \frac{\partial \psi_{1}}{\partial s}(0, t)\right) d t=0 .
\end{aligned}
$$

As condition (40) is valid for any subinterval $\left[t_{a}, t_{b}\right] \subseteq\left[t_{1}, t_{2}\right]$, the only possibility of this happening is

$$
\begin{aligned}
\sum_{l=1}^{n} \partial_{l} \mathbf{H} \cdot\left(\frac{\partial L_{l}[q]^{m}(t)}{\partial t} \frac{\partial \psi_{1}}{\partial s}(0, t)+\sum_{i=0}^{m} \frac{\partial L_{l}[q]^{m}(t)}{\partial q^{(i)}(t)}\right. & \left.\frac{\partial}{\partial s} \frac{d^{i} \psi_{2}(s, q(t))}{d \psi_{1}^{i}(s, t)}\right|_{s=0} \\
& \left.+L_{l}[q]^{m}(t) \frac{d}{d t} \frac{\partial \psi_{1}}{\partial s}(0, t)\right)=0
\end{aligned}
$$

Using (36)-(37) and (39), and the fact $d / d t$ acts on variable $t$ and $\partial / \partial s$ on variable $s$, and $\psi_{i}(s, q)$ is a smooth family in $\mathbb{W}^{m, p}(\mathbb{R})$ (cf. Definition 4.1 ), we have

$$
\left.\frac{\partial}{\partial s}\left[\frac{d \psi_{i}}{d t}(s, \cdot)\right]\right|_{s=0}=\frac{d}{d t} \frac{\partial \psi_{i}}{\partial s}(0, \cdot)
$$


with $i=1,2$, and

$$
\left\{\begin{array}{l}
\left.\frac{\partial}{\partial s}\left(\frac{d \psi_{2}(s, q(t))}{d \psi_{1}(s, t)}\right)\right|_{s=0}=\frac{d}{d t} \frac{\partial \psi_{2}}{\partial s}(0, q(t))-\dot{q}(t) \frac{d}{d t} \frac{\partial \psi_{1}}{\partial s}(0, t) \\
\left.\frac{\partial}{\partial s}\left(\frac{d^{i} \psi_{2}(s, q(t))}{d \psi_{1}^{i}(s, t)}\right)\right|_{s=0} \\
=\left.\frac{d}{d t} \frac{\partial}{\partial s}\left(\frac{d^{i-1} \psi_{2}(s, q(t))}{d \psi_{1}^{i-1}(s, t)}\right)\right|_{s=0}-q^{(i)}(t) \frac{d}{d t} \frac{\partial \psi_{1}}{\partial s}(0, t), i=2, \ldots, m .
\end{array}\right.
$$

Substituting the system (42) into equation (41), we obtain condition (38).

Definition 4.4 (Constant of motion/conservation law). We say that a equation $C[q]^{m}(t)$ is a constant of motion if

$$
\frac{d}{d t} C[q]^{m}(t)=0, \forall t \in\left[t_{1}, t_{2}\right]
$$

along all the extremals $q(\cdot)$ (cf. Definition 3.3). The equality (43) is then a conservation law.

In physics, a conservation law asserts that a particular measurable property $P$ of an isolated physical system does not change as the system evolves. Now, we are able to prove the most important result of this section.

Theorem 4.5 (Higher-order Noether's theorem). If functional (1) is invariant in the sense of Definition 4.2, then the equation $C[q]^{m}(t)$ defined by

$$
\sum_{l=0}^{n} \partial_{l} \mathbf{H} \cdot\left[\sum_{i=1}^{m} \varphi^{i} \rho^{i-1}(t)+\left(L_{l}[q]^{m}(t)-\sum_{i=1}^{m} \varphi^{i} q^{(i)}(t)\right) \frac{\partial \psi_{1}}{\partial s}(0, t)\right]
$$

is a constant of motion (cf. Definition 4.4).

Proof. Equation (44) follows by direct calculations:

$$
\begin{array}{r}
\sum_{l=0}^{n} \partial_{l} \mathbf{H} \cdot\left[\sum_{i=1}^{m} \varphi^{i} \rho^{i-1}(t)+\left(L_{l}[q]^{m}(t)-\sum_{i=1}^{m} \varphi^{i} q^{(i)}(t)\right) \frac{\partial \psi_{1}}{\partial s}(0, t)\right] \\
=\sum_{l=0}^{n} \partial_{l} \mathbf{H} \cdot\left[\varphi^{1} \rho^{0}(t)+\sum_{i=2}^{m} \varphi^{i} \rho^{i-1}(t)+\left(L_{l}[q]^{m}(t)-\sum_{i=1}^{m} \varphi^{i} q^{(i)}(t)\right) \frac{\partial \psi_{1}}{\partial s}(0, t)\right] .
\end{array}
$$


Differentiating (45) with respect to $t$, yields

$$
\begin{aligned}
& \sum_{l=0}^{n} \partial_{l} \mathbf{H} \cdot\left[\rho^{0}(t) \frac{d}{d t} \varphi^{1}+\varphi^{1} \frac{d}{d t} \rho^{0}(t)+\sum_{i=2}^{m}\left(\rho^{i-1}(t) \frac{d}{d t} \varphi^{i}+\varphi^{i} \frac{d}{d t} \rho^{i-1}(t)\right)\right. \\
& \left.\quad+\frac{\partial \psi_{1}}{\partial s}(0, t) \frac{d}{d t}\left(L_{l}[q]^{m}(t)-\sum_{j=i}^{m} \varphi_{1}^{i} q^{(i)}(t)\right)+\left(L_{l}[q]^{m}(t)-\sum_{j=i}^{m} \varphi_{1}^{i} q^{(i)}(t)\right) \frac{d}{d t} \frac{\partial \psi_{1}}{\partial s}(0, t)\right] .
\end{aligned}
$$

Using the higher-order Euler-Lagrange equation (14), the higher-order DuBoisReymond condition (21), and relations (19) and (39) in (46), one gets

$$
\begin{gathered}
\sum_{l=0}^{n} \partial_{l} \mathbf{H} \cdot\left[\partial_{2} L_{l}[q]^{m}(t) \frac{\partial \psi_{2}}{\partial s}(0, q(t))+\varphi^{1}\left(\rho^{1}(t)+q^{(1)}(t) \frac{d}{d t} \frac{\partial \psi_{1}}{\partial}(0, t)\right)\right. \\
+\sum_{i=2}^{m}\left\{\left(\partial_{i+1} L_{l}[q]^{m}(t)-\varphi^{i-1}\right) \rho^{i-1}(t)+\varphi^{i}\left(\rho^{i}(t)+q^{(i)}(t) \frac{d}{d t} \frac{\partial \psi_{1}}{\partial s}(0, t)\right)\right\} \\
\left.+\partial_{1} L_{l}[q]^{m}(t) \frac{\partial \psi_{1}}{\partial s}(0, t)+\left(L_{l}[q]^{m}(t)-\sum_{i=1}^{m} \varphi^{i} q^{(j)}(t)\right) \frac{d}{d t} \frac{\partial \psi_{1}}{\partial s}(0, t)\right] \\
=\sum_{l=0}^{n} \partial_{l} \mathbf{H} \cdot\left[\partial_{1} L_{l}[q]^{m}(t) \frac{\partial \psi_{1}}{\partial s}(0, t)+L_{l}[q]^{m}(t) \frac{d}{d t} \frac{\partial \psi_{1}}{\partial s}(0, t)+\partial_{2} L_{l}[q]^{m}(t) \frac{\partial \psi_{2}}{\partial s}(0, q(t))\right. \\
+\varphi^{1}\left(\rho^{1}(t)+q^{(1)}(t) \frac{d}{d t} \frac{\partial \psi_{1}}{\partial s}(0, t)\right)-\varphi^{1} \rho^{1}(t) \\
\left.-\varphi^{1} q^{(1)}(t) \frac{d}{d t} \frac{\partial \psi_{1}}{\partial s}(0, t)+\varphi^{m} \rho^{m}(t)+\sum_{i=2}^{m} \partial_{i+1} L_{l}[q]^{m}(t) \cdot \rho^{i-1}(t)\right]
\end{gathered}
$$

Simplification of (47) leads to the necessary condition of invariance (38).

Corollary 4.6. Suppose that the functional $\mathcal{J}_{n}^{1}$ ( cf. 3) is invariant in the sense of Definition 4.2 with $m=1$, then the quantity $C[q]^{1}(t)$ defined by

$$
\begin{aligned}
\sum_{l=0}^{n} \partial_{l} \mathbf{H} \cdot\left[\frac{\partial \psi_{2}}{\partial s}\right. & (0, q(t)) \partial_{3} L_{l}\left(t, q(t), q^{(1)}(t)\right) \\
& \left.+\frac{\partial \psi_{1}}{\partial s}(0, t)\left(L_{l}\left(t, q(t), q^{(1)}(t)\right)-q^{(1)}(t) \partial_{3} L_{l}\left(t, q(t), q^{(1)}(t)\right)\right)\right]
\end{aligned}
$$

is a constant of motion. 
Proof. Using relations (19) and (39), we have

$$
\begin{gathered}
\sum_{i=1}^{m} \varphi^{i} \rho^{i-1}(t)=\frac{\partial \psi_{2}}{\partial}(0, q(t)) \sum_{j=0}^{m-1}(-1)^{j} \frac{d^{j}}{d t^{j}} \frac{\partial L_{l}[q]^{m}(t)}{\partial q^{(j+1)}(t)} \\
+\sum_{i=2}^{m}\left\{( \sum _ { j = 0 } ^ { m - i } ( - 1 ) ^ { j } \frac { d ^ { j } } { d t ^ { j } } \frac { \partial L _ { l } [ q ] ^ { m } ( t ) } { \partial q ^ { ( j + 1 ) } ( t ) } ) \left(\frac{d^{i-1}}{d t^{i-1}} \frac{\partial \psi_{2}}{\partial s}(0, q(t))-\dot{q}(t) \frac{d^{i-1}}{d t^{i-1}} \frac{\partial \psi_{1}}{\partial s}(0, t)\right.\right. \\
\left.\left.-\sum_{j=1}^{i-2}\left(\begin{array}{c}
i-1 \\
i-j-1
\end{array}\right) q^{(j+1)}(t) \frac{d^{i-j-1}}{d t^{i-j-1}} \frac{\partial \psi_{1}}{\partial s}(0, t)\right)\right\}
\end{gathered}
$$

and

$$
\begin{aligned}
& \left(L[q]^{m}(t)-\sum_{i=1}^{m} \varphi^{i} q^{(i)}(t)\right) \frac{\partial \psi_{1}}{\partial s}(0, t) \\
= & \left\{L_{l}[q]^{m}(t)-\sum_{i=1}^{m}\left(\sum_{j=0}^{m-i}(-1)^{j} \frac{d^{j}}{d t^{j}} \frac{\partial L[q]^{m}(t)}{\partial q^{(j+1)}(t)}\right) q^{(i)}(t)\right\} \frac{\partial \psi_{1}}{\partial s}(0, t) .
\end{aligned}
$$

For $m=1$, from (49)-(50) we have

$$
\begin{aligned}
& \frac{\partial \psi_{2}}{\partial s}(0, q(t)) \cdot(-1)^{0} \frac{d^{0}}{d t^{0}} \frac{\partial L[q]^{m}(t)}{\partial q^{(1)}(t)}+\left\{L[q]^{1}(t)-(-1)^{0} \frac{d^{0}}{d t^{0}} \frac{\partial L[q]^{1}(t)}{\partial q^{(1)}(t)} \cdot q^{(1)}(t)\right\} \frac{\partial \psi_{1}}{\partial s}(0, t) \\
= & \frac{\partial \psi_{2}}{\partial s}(0, q(t)) \partial_{3} L_{l}\left(t, q(t), q^{(1)}(t)\right)+\frac{\partial \psi_{1}}{\partial s}(0, t)\left(L_{l}\left(t, q(t), q^{(1)}(t)\right)-q^{(1)}(t) \partial_{3} L_{l}\left(t, q(t), q^{(1)}(t)\right)\right) .
\end{aligned}
$$

\section{Acknowledgment}

J. Vanterler acknowledges the financial support of a PNPD-CAPES ( 88882.305834/2018-01) scholarship of the Postgraduate Program in Applied Mathematics of IMECC-Unicamp.

\section{References}

[1] Almeida, R., and Delfim F. M. Torres. "Calculus of variations with fractional derivatives and fractional integrals." Appl. Math. Lett. 22.12 (2009): 1816-1820.

[2] Almeida, R., and Delfim F. M. Torres. "Necessary and sufficient conditions for the fractional calculus of variations with Caputo derivatives." Commun. Nonlinear Sci. Numer. Simul. 16.3 (2011): 1490-1500.

[3] Atanacković, T. M., S. Konjik, and S. Pilipović. "Variational problems with fractional derivatives: Euler-Lagrange equations." J. Phys. A: Math. Theor. 41.9 (2008): 095201.

[4] Bartosiewicz, Z., Natália Martins, and Delfim FM Torres. "The second Euler-Lagrange equation of variational calculus on time scales." European Journal of Control 17.1 (2011): $9-18$. 
[5] Brezis, H., Functional analysis, Sobolev spaces and partial differential equations, Universitext, Springer, New York, 2011.

[6] Brunt, B. Van. The calculus of variations, Universitext, SpringerVerlag, New York, 2004.

[7] Bliss, G. A.. "The problem of Lagrange in the calculus of variations." Amer. J. Math. 52.4 (1930): 673-744.

[8] Castillo, E., A. Luceno, and P. Pedregal. "Composition functionals in calculus of variations: application to products and quotients." Math. Model. Meth. Appl. Sci. 18.01 (2008): 47-75.

[9] Castilo, E., A.Luceño and P. Pedregal, Composition fuctionals in calculus of variations: applications to products and quocients, Math. Models Meth. Appl. Sci. 18 460-481, 2008.

[10] Christyanti, R. Dwi, R. B. E. Wibowo, and A. R. Alghofari. "Existence and Uniqueness Solution of Euler-Lagrange Equation in Sobolev Space $W^{1, p}(\Omega)$ with Gateaux Derivative." Inter. J. Math. Anal. 8.40 (2014): 1987-1998.

[11] Clarke, F. H., and R. B. Vinter. "A regularity theory for variational problems with higher order derivatives." Trans. Amer. Math. Soc. 320.1 (1990): 227-251.

[12] Clarke, F. H., and Richard B. Vinter. "Existence and regularity in the small in the calculus of variations." J. Diff. Equ. 59.3 (1985): 336-354.

[13] Clarke, F. H. "The Euler-Lagrange differential inclusion.” J. Diff. Equ. 19.1 (1975): 80-90.

[14] Dacorogna, B., Introduction to the Calculus of Variations, Imperial College Press, London, 2004

[15] Frederico, G. S. F., and Delfim F. M. Torres. "Noether's symmetry Theorem for variational and optimal control problems with time delay." Numer. Algebra, Control Opt. 2.3 (2012): 619.

[16] Frederico, G. S. F., and Delfim F. M. Torres. "Fractional Noether's theorem in the Riesz-Caputo sense." Appl. Math. Comput. 217.3 (2010): 1023-1033.

[17] Frederico, G. S. F., T. Odzijewicz, and Delfim F. M. Torres. "Noether's theorem for nonsmooth extremals of variational problems with time delay." Applicable Anal. 93.1 (2014): 153-170.

[18] Frederico, G. S. F., and Matheus J. Lazo. "Fractional Noether's theorem with classical and Caputo derivatives: constants of motion for non-conservative systems." Nonlinear Dyn. 85.2 (2016): 839-851.

[19] Frederico, G. S. F., and Delfim F. M. Torres. "A formulation of Noether's theorem for fractional problems of the calculus of variations." J. Math. Anal. Appl. 334.2 (2007): 834-846.

[20] Gel'fand, I.M., and Fomin, S.V., Calculus of Variations, Prentice-Hall, Inc., Englewood Cliffs, N.J., 1963.

[21] Glasgow, Larry A. Applied Mathematics for Science and Engineering. Wiley, 2014.

[22] Guzowska, M., A. B. Malinowska, and M. R. S. Ammi. "Calculus of variations on time scales: applications to economic models." Adv. Diff. Equ. 2015.1 (2015): 203.

[23] Herzallah, M. A. E., and D. Baleanu. "Fractional euler-lagrange equations revisited." Nonlinear Dyn. 69.3 (2012): 977-982.

[24] Hildebrandt, S., and Tromba, A., Mathematics and Optimal Form, Scientific American Books, New York, 1985.

[25] Jost, J. and X. Li-Jost, Calculus of variations, Cambridge Studies in Advanced Mathematics, 64, Cambridge Univ. Press, Cambridge, 1998.

[26] Komkov, V.. "A dual form of Noether's theorem with applications to continuum mechanics." J. Math. Anal. Appl. 75.1 (1980): 251-269.

[27] Kot, M., A First Course in the Calculus of Variations, American Mathematical Society, Providence, R.I., 2014.

[28] Lazo, M. J., and Delfim F. M. Torres. "The DuBois-Reymond fundamental lemma of the fractional calculus of variations and an Euler-Lagrange equation involving only derivatives of Caputo." J. Opt. Theory Appl. 156.1 (2013): 56-67.

[29] Lu, G., and B. Ou, A Poincaré inequality on $\mathbb{R}^{n}$ and its application to potential fluid flows in space, Comm. Appl. Nonlinear Anal. 12 (1), 1—24, 2005. 
[30] Martins, N., and Delfim F. M. Torres. "Calculus of variations on time scales with nabla derivatives." Nonlinear Analysis: Theory, Methods \& Applications 71.12 (2009): e763e773.

[31] Malinowska, A. B., and Delfim F. M. Torres. "Euler-Lagrange equations for composition functionals in calculus of variations on time scales." Disc. Cont. Dyn. Sys.-A 29.2 (2011): 577.

[32] Malinowska, A. B., and Delfim F. M. Torres. Introduction to the fractional calculus of variations. World Scientific Publishing Company, 2012.

[33] McShane, E. J. "The Du Bois-Reymond relation in the calculus of variations." Math. Annalen 109.1 (1934): 746-755.

[34] Noether, E., Invariante Variationsprobleme, Nachr. König. Gesell. Wissen. Göttingen, Math.-Phys. Kl., 235-257, 1918.

[35] Torres, Delfim F. M. "Proper extensions of Noether's symmetry theorem for nonsmooth extremals of the calculus of variations." Commun. Pure Appl. Anal. 3.3 (2004): 491.

[36] Troutman, J. L., Variational calculus and optimal control, second edition, Undergraduate Texts in Mathematics, Springer, New York, 1996.

[37] Santos, Simão P. S., N. Martins, and Delfim F. M. Torres. "Noether's theorem for higherorder variational problems of Herglotz type." Conference Publications. Vol. 2015. No. special. Amer. Inst. Math. Sci., 2015.

[38] Santos, S. P. S., N. Martins, and Delfim F. M. Torres. "Variational problems of Herglotz type with time delay: DuBois-Reymond condition and Noether's first theorem." Disc. Cont. Dyn. Sys.-A 35.9 (2015): 4593.

[39] Odzijewicz, T., and Delfim F. M. Torres. "Calculus of Variations with Classical and Fractional Derivatives." Math. Balkanica New Series 26.1-2 (2012): 191-202.

[40] Mouchet, A.. "Applications of Noether conservation theorem to Hamiltonian systems." Annals of Phys. 372 (2016): 260-282.

[41] Santos, S. P. S., N. Martins, and Delfim F. M. Torres. "Noether currents for higher-order variational problems of Herglotz type with time delay." Disc. Cont. Dyn. Sys.-S 11.1 (2018): 91.

[42] Tavel, M. "Application of Noether's theorem to the transport equation." Transport Theory and Statistical Physics 1.4 (1971): 271-285. 